\section{Potentially inappropriate use of antipsychotics in community- dwelling adults with dementia more common in those with low income}

doi:10.1136/eb-2012-101180

\section{QUESTION}

Question: Does both essential and potentially inappropriate use of antipsychotics vary across income groups?

Population: Essential antipsychotic use was assessed in 11417 adults (aged 19-64 years, 60\% men, 84\% urban) with a diagnosis of schizophrenia (international classification of diseases (ICD-9), ICD-10; 27\% hospitalised for schizophrenia). Potentially inappropriate use of antipsychotics was assessed in 33633 older adults (aged 65 years and older, 60\% women; $23 \%$ in long-term care facilities) with dementia (DSM-IV) and no diagnosis of schizophrenia or bipolar disorder. People living in British Columbia (BC) for less than 9 months were excluded, as were people with incomplete data, or living in areas with a high proportion of non-fee for service claims.

Setting: British Columbia, Canada; 2004-2005.

Assessment: Physician and hospital diagnostic records were searched for people with diagnoses of schizophrenia and dementia in 2004 or 2005 . Hospitalisation for schizophrenia was taken as a crude measure of severity. Data on filled prescriptions for antipsychotics in 2005 were obtained from the PharmaNet database of prescriptions filled in community pharmacies in BC. For $83 \%$ of the sample, income quintile was derived from the BC Ministry of Health Services which records income data for people registered with the incomebased drug benefit programme. For the remaining $17 \%$, average household tax returns from census dissemination areas (400-700 people) were used. Overall health status was assessed using Aggregated Diagnostic Groupings (ADGs). Sex, age and urban or non-urban residence status was recorded. Income-related disparities in antipsychotic use were assessed using multivariable logistic regression analyses (adjusted for age, gender, health status, residence and hospitalisation for schizophrenia if relevant). The highest income quintile was used as the reference group for calculation of ORs.
Outcomes: Antipsychotic use; long-term antipsychotic use (defined as receiving a 180-day supply for essential use and a 90-day supply for inappropriate use).

\author{
METHODS \\ Design: Retrospective cohort study.
}

\section{MAIN RESULTS}

Essential antipsychotic use: $85 \%$ of participants had filled a prescription for antipsychotics in 2005, with $71 \%$ having longterm use. The highest and lowest income groups had about the same odds for essential use. The third and fourth quintiles had greater odds of use then the highest quintile. Findings were similar for long-term use (see Webextra table). Potentially inappropriate use: Overall, $23 \%$ of adults with dementia received antipsychotics, with $14 \%$ taking antipsychotics long term. Among community-dwelling adults, any antipsychotic use and long-term use were more likely in the lowest income quintile than in the highest income quintile (see Webextra table). There were no significant differences in antipsychotic use among income quintiles in people in long-term care. People with dementia in long-term care were more likely to receive antipsychotics compared with people living in the community (56\% vs 13\%; OR 9.31, 95\% CI 8.75 to 9.91).

\section{CONCLUSIONS}

Potentially inappropriate use of antipsychotics is more prevalent in older adults with dementia from low-income households than those from high-income households. Potentially, inappropriate antipsychotic use is also more prevalent in those in long-term care than those living in the community.

\section{ABSTRACTED FROM}

Puyat JH, Law MR, Wong ST, et al. The essential and potentially inappropriate use of antipsychotics across income groups: an analysis of linked administrative data. Can J Psychiatry 2012;57:488-95.

Correspondence to: Puyat JH, School of Population and Public Health, University of British Columbia, 201-2206 East Mall, Vancouver, Canada BC V6T 1Z3; jpuyat@ interchange.ubc.ca

Sources of funding: BCMOHS, Canadian Health Services Research Foundation; Alberta Heritage Foundation for Medical Research, Canadian Institutes of Health Research.

- Additional material is published online only. To view please visit the journal online (http://dx.doi.org/10.1136/eb-2012-101180).
Pis yat and colleagues examine the relationship between antipsychotic use and household income among community-dwelling and institutionalised patients. Based on analyses of linked health, pharmaceutical use and income data from British Columbia from 2004 to 2005, they found income-related differences in essential antipsychotic use for schizophrenia or bipolar disorder, and inappropriate antipsychotic use among community-dwelling older adults. In both cases, low-income individuals were at higher risk of poorer prescribing. Given the paucity of data regarding income-related effects on inappropriate medication use, these data will likely receive attention. However, these provocative findings should be interpreted in proper context. First, the authors estimate antipsychotic prescribing in long-term care to be $56 \%$, a finding that is not replicated in the literature. A point-prevalence study of Ontario nursing homes reported that $32.4 \%$ of residents received antipsychotics in 2003. ${ }^{1}$ Most published studies report similar findings. Second, Mor et $a l^{2}$ report that low resource nursing homes serve predominantly poor communities and have higher levels of antipsychotic use. Complicated interactions between community poverty and healthcare facility resources likely explain much of the relationship between prescribing and household income in community mental health and primary care clinics as well. These factors are also likely to be related to 'prescribing cultures', ${ }^{3}$ institutional forces that drive prescribing, independent of clinical appropriateness. This phenomenon defines the patient risk factor as exposure to healthcare facilities with high levels of inappropriate prescribing. While primarily described in long-term care, this phenomenon likely exists in community care settings as well. Taken together, complex relationships between household income, community poverty and healthcare facility resources likely contribute to inappropriate care. Improvements in prescribing need to focus beyond payment and public medication coverage to also address the context of care available to impoverished patients.

\section{Jennifer Tjia}

Department of Medicine, University of Massachusetts Medical School, Worcester, Massachusetts, USA

\section{Competing interests None.}

\section{REFERENCES}

1. Rochon PA, Stukel TA, Bronskill SE, et al. Variation in nursing home antipsychotic prescribing rates. Arch Intern Med 2007;167:676-83.

2. Mor V, Zinn J, Angelelli J, et al. Driven to tiers: socioeconomic and racial disparities in the quality of nursing home care. Milbank 0 2004;82:227-56.

3. Tjia J, Gurwitz JH, Briesacher BA. The challenge of changing nursing home prescribing culture. $A m \mathrm{~J}$ Geriatr Pharmacotherapy 2012;10:37-46. 